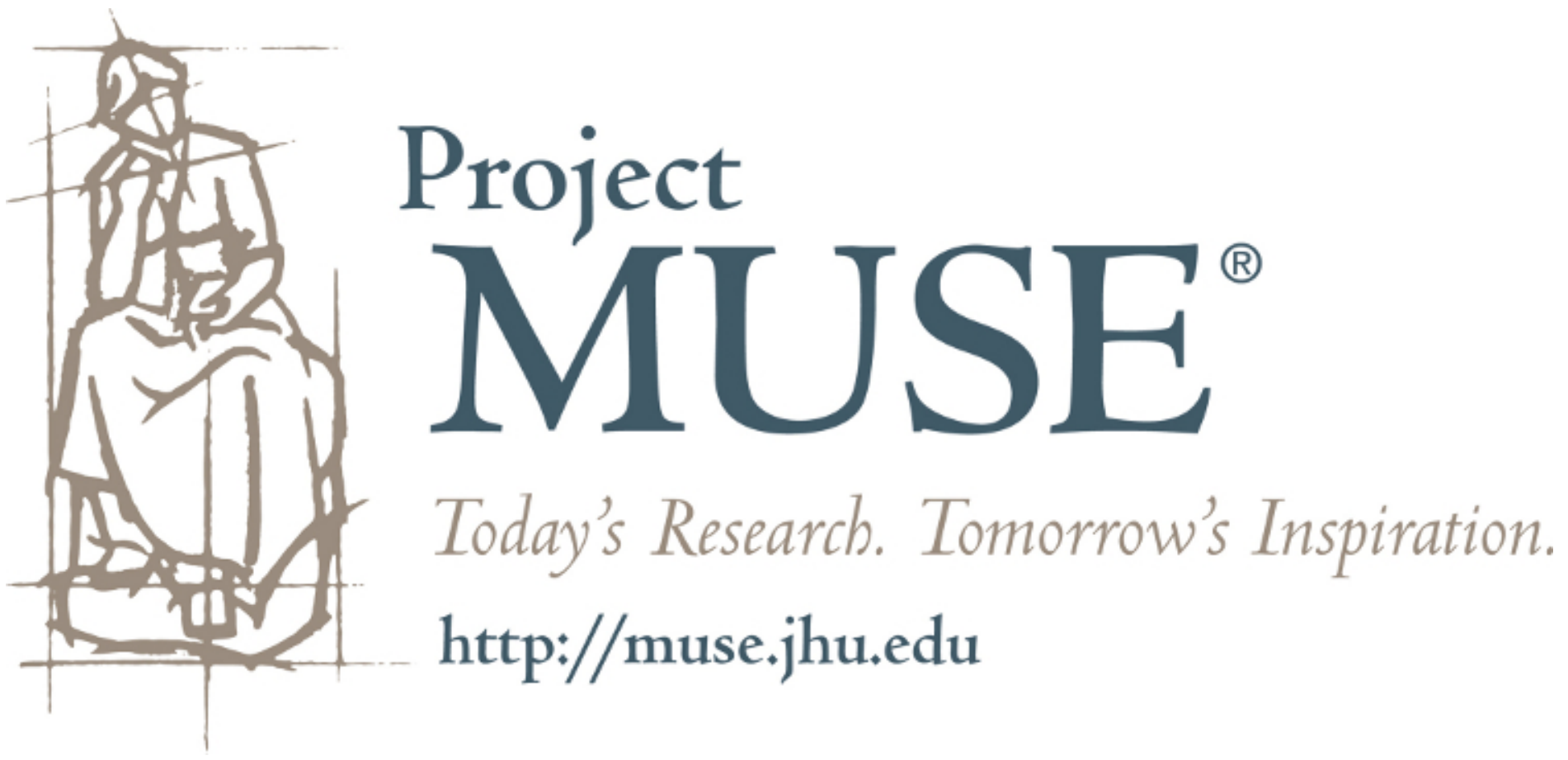




\section{Dance Jockey: Performing Electronic Music by Dancing}

\section{Yago de Quay, Ståle Skogstad and Alexander Jensenius}

$\mathrm{T}_{\mathrm{n}}$ he use of sensors for converting environmental data into information for performances is helping us to rethink the way electronic music is composed and performed. Motion-capture technology-the process of acquiring data in a computer about human limbs and large-scale body movements-was previously limited mostly to the field of computer animation. It has been slowly migrating toward the performing arts, fostering collaboration between artists and scientists. In an example using biofeedback, Pamela $\mathrm{Z}$ developed a system allowing interplay between her voice and a gesture-based MIDI controller called BodySynth that translates electrical signals generated by muscle contractions into MIDI commands [1]. The New York dance company Troika Ranch has been known to use a wireless suit of up to eight bend sensors to manipulate visual and sonic elements [2]. The Palindrome dance company has been sonically and visually augmenting their performances using their own EyeCon motion-tracking software [3]. Dobrian and Bevillacqua experiment with the Vicon 8 optical motion-capture system to control music and lighting [4]. Goto and Suzuki [5] use a 12-bend-sensor body suit mapped to granular synthesis parameters and visuals. Inspired by Japanese anime and manga comics, Bahn and Hahn [6] composed a solo performance called Pikapika which has Hahn, the dancer, holding an accelerometer and a force-sensitive resistor that are used to control various sound effects.

There have been few music performances using the Xsens MVN motion-capture suit. The musician Praga Khan has used it to control visuals [7]. Ghent and McGill Universities have tested mapping strategies and gait recognition, but using only a few sensors, not the full MVN system [8, 9].

As Karlheinz Brandenburg, director of the Fraunhofer Institute and co-creator of MP3, has said, "We will see new forms of listening to music and creating music. . . . Interactivity will blur the lines between listening, creating music and computer games" [10]. However, most of the systems described above have had limited real-world application, and their designers' goals were merely to "sonify" the data derived from the sensors [11].

Dance Jockey, composed of two of us (co-authors Yago de Quay and Ståle Skogstad), refocuses sensors from simplistic Yago de Quay (sound designer), Faculty of Engineering, University of Porto, Rua
Dr. Roberto Frias, s/n, 4200-465 Porto, Portugal. E-mail: <yagodequay@gmail.com>.

Ståle Skogstad (researcher), fourMs group—Music, Mind, Motion, Machines, University of Oslo, Department of Informatics, 0316 Oslo, Norway. E-mail: <savskogs@ifi.uio.no>.

Alexander Jensenius (researcher), fourMs group—Music, Mind, Motion, Machines University of Oslo, Department of Musicology, 0371 Oslo, Norway. E-mail: <a.r.jensenius@ imviuio.no>.

See $<$ mitpressjournals.org/toc/lmj/-/21 > for supplemental files (such as audio and video) related to this issue of $\mathrm{LMJ}$ and accompanying $\mathrm{CD}$. action-to-sound function to contextualized aesthetic and dramatic expression. This exploratory work has been shown publicly as work in progress, with the hope of discovering new possibilities afforded by making music through dance. De Quay is responsible for performing onstage (Fig. 1) and composing the music; Skogstad is responsible for the hardware and software; we decide choreography and artistic direction together. The music and choreography are ever changing, and our main goal is to express and sonically improvise various actions happening onstage, fleshing out the stories of various characters as we travel through a world of sounds.

\section{THE SUIT}

The Xsens MVN suit consists of 17 small sensors worn over the performer's clothes, tracking all limbs in 3D space. It offers the following advantages: (1) it is lightweight and portable; (2) its setup time is quick (15 minutes); (3) it allows communication with third-party programs; and (4) it is wireless.

\section{THE SCRIPT}

Developing the performance has been an iterative process, with music, choreography and software evolving side by side. Our initial performances were rigid and simple-they did not allow for many changes in the script and presented only very obvious relationships between sound and movement. As we gained experience, we started reaching a point where the acts of composing and performing were indistinguishable, helping to spawn more complex and richer interactions.

\section{THE SOUND}

Sounds oscillate between theatrical sound effects and musical compositions. The former sonically augment the actions that are happening onstage, such as steps, noises by objects and machines. Musical compositions focus on the instrumentation of the body, having as the main priority music coherence and rhythm reflected by the scripts' sounds and choreography. These range from simple wind chimes to full songs in which the beat, synth and voices are controlled simultaneously with different body parts. 


\section{THE SOFTWARE}

The Jamoma framework is a community-driven software built for the MAX/ MSP programming environment, which offers plug-and-play modules ideal for flexible art performances. This performance uses three modules-cues, actions and transitions-modeled after the finite-state machine. Cues are sequenced before performance and summon a set of actions that harbor a unique relationship between the Xsens MVN output and MIDI or open sound control (OSC) values. For example, an action could be mapping hand height measured in centimeters to a $0-127$ scale. Transitions encapsulate specific conditions that, if met by the Xsens output, will hop from the current cue to the next. Audio is generated in Ableton Live 8 , chosen because of its MIDI and OSC protocols and robust live performance.

\section{CONCLUSION}

We have staged Dance Jockey four times in Oslo, Norway, and once in Portugal between September 2010 and May 2011, attracting more than 1,000 attendees. The public has reacted with interest to the clear relationship between body movement and sound, which enables better understanding and appreciation of digital art performances. Moreover, the performance encourages a look at the difference between dancing along to music and making music by dancing.

This project grew out of our interest in understanding the processes of innovation and technology diffusion in artistic performances. Music and technology coevolve, both shaping and being shaped by human expression and creativity. The variety and intricacy of these recombination processes contribute profoundly to the current diversity of performance structures and meanings within the arts.

Fig. 1. The dancer, Yago de Quay, controlling sounds using the Xsens MVN system in Dance Jockey during its third performance at the VERDIKT Conference in Oslo, Norway. (Photo @ Alexander Jensenius)

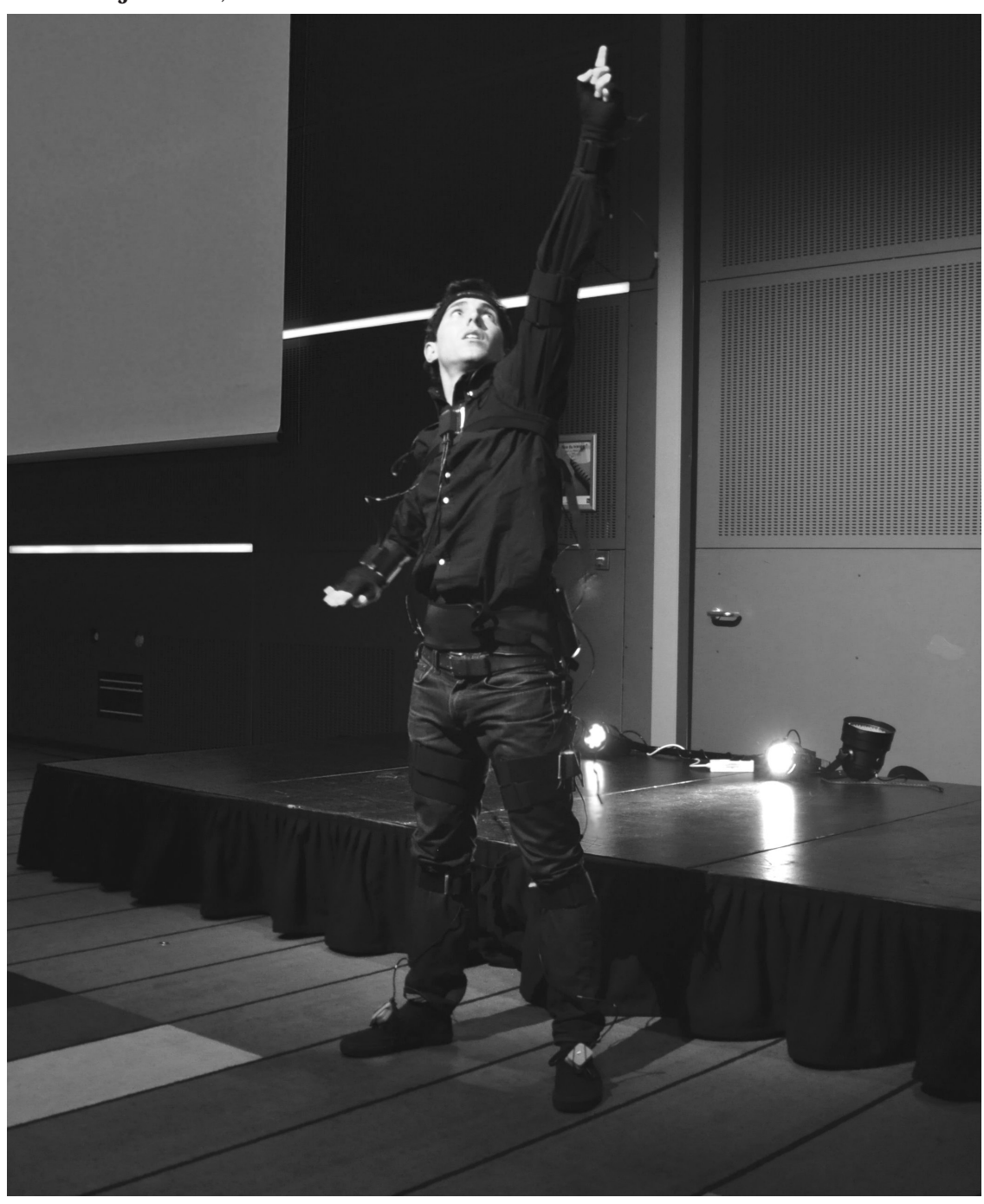

\section{References and Notes}

1. BodySynth (cited April 2011]; Available at: <www. synthzone.com/bsynth.h) ml>.

2. Troika Ranch (cited 2011 April); Available at: $<$ www.troikaranch.org $>$.

3. Palindrome (cited 2010 June); Available at: $<$ www. palindrome.de $>$.

4. C. Dobrian and F. Bevilacqua, "Gestural Control of Music: Using the Vicon 8 Motion Capture System," in Proceedings of the 2003 Conference on New Interfaces for Musical Expression, Montreal (National Univ. of Singapore, 2003) pp. 161-163.

5. S. Goto and T. Suzuki, "The Case Study of Application of Advanced Gesture Interface and Mapping Interface: Virtual Musical Instrument 'Le SuperPolm' and Gesture Controller 'BodySuit,'” in Proceedings of the 2004 Conference on New Interfaces for Musical Expression, Hamamatsu, Shizuoka, Japan (National Univ. of Singapore, 2004) pp. 207-208.

6. C. Bahn, T. Hahn and D. Trueman, "Physicality and Feedback: A Focus on the Body in the Performance of Electronic Music," in International Computer Music Conference (Havana, Cuba, 2001).

7. N. Collins et al., "Musical Exoskeletons: Experiments with a Motion Capture Suit," in New Interfaces for Musical Expression (NIME) (Sydney, Australia, 2010).

8. P.-J. Maes et al., "From Expressive Gesture to Sound: The Development of an Embodied Mapping Trajectory inside a Musical Interface," Journal on ping Trajectory inside a Musical Interface," Journal on
Multimodal User Interfaces 31, Nos. 1-2, 67-78 (2010).

9. A.J. Malozemoff and P. Depalle, MUMT 502 Project Report: Gait Recognition Using Accelerometers and Sound (Montreal: McGill University, 2009).

10. K. Brandenburg quoted in Maria Popova, "The Big Question: The Next 10 Years of the Music Industry," Wired (14 May 2010).

11. Bahn et al. [6]; G. Bertini, M. Magrini and L. Tarabella, "An Interactive Musical Exhibit Based on Infrared Sensors," in R. Kronland-Martinet, T. Voinier and S. Ystad, eds., Computer Music Modeling and Retrieval (Berlin/Heidelberg: Springer, 2006) pp-100; C. Salter, M. Baalman and D. Moody-Grigsby, "Between Mapping, Sonification and Composition: Responsive Audio Environments in Live Performance," in Kronland-Martinet et al. pp. 246-262; J. Futrelle and S. Downie, "Interdisciplinary Research Issues in Music Information Retrieval: ISMIR 2002," Journal of New Music Research 32, No. 2, $121-131$ (2003).

Manuscript received 1 January 2011.

Yago de Quay is an interactive media artist, musician and researcher based in Porto and Oslo. His numerous installations and performances focus on user participation contributing to modify the art piece itself. They always have a strong sonic component and combine technologies to help create new modes of expression.

Ståle Skogstad is a Ph.D. student in the fourMs group at the Department of Informatics at the University of Oslo and holds a Master's degree in Computer Science. His research is focused on using real-time full-body motion-capture technology for musical interaction. The main goal is to explore the potentials of these technologies for musical applications.

Alexander Refsum Jensenius is a music researcher and research musician working in the fields of embodied music cognition and new instruments for musical expression at the University of Oslo and Norwegian Academy of Music. 